\title{
Diagnostic Yield and Therapeutic Impact of Face and Neck Imaging in Patients Referred with Otalgia without Clinically Overt Disease
}

\author{
DE. Ainsworth, (DI. Pai, (D). Kathirgamanathan, and (DS.E.J. Connor
}

\begin{abstract}
BACKGROUND AND PURPOSE: Otalgia may be secondary to serious pathology, such as upper aerodigestive tract malignancies, and CT or MR imaging of the skull base, face, and neck is often performed to detect clinically occult lesions. The diagnostic yield, management impact, and therapeutic impact of imaging in this clinical scenario, however, have yet to be elucidated.
\end{abstract}

MATERIALS AND METHODS: CT and MR imaging in patients who presented with otalgia without clinically overt disease was retrospectively analyzed from a single center over a 9-year period. The cohort was subdivided into groups, depending on the presence of additional symptoms and a history of head and neck cancer. Relevant diagnostic outcome findings were categorized, and the diagnostic yield and impact of imaging on management and therapy were calculated for each group.

RESULTS: In our study cohort of 235 patients, the diagnostic yield of imaging for otalgia, with or without other symptoms, in patients who lacked a history of head and neck cancer was negligible for upper aerodigestive tract malignancy (1\%), abnormalities related to otalgia ( $2 \%)$, and other moderate or major findings $(2 \%)$. Although equivocal or unimportant findings occasionally resulted in additional investigations, the therapeutic impact was also very low (2\%). The diagnostic yield for upper aerodigestive tract malignancy (34\%) and therapeutic impact increased (34\%) when there was a history of head and neck cancer.

CONCLUSIONS: The diagnostic yield and therapeutic impact of imaging for otalgia without clinically overt disease are very low, unless there is a history of head and neck cancer.

ABBREVIATIONS: FNE = fibreoptic nasendoscopy; UAT = upper aerodigestive tract

$\mathrm{T}$ he causes of otalgia (earache) are diverse and can be categorized as primary and secondary. Primary causes of otalgia are pathologies that affect the ear itself, ${ }^{1}$ with common causes that include otitis externa, otitis media, ${ }^{2}$ trauma, and foreign bodies. Primary otalgia often presents with overt clinical signs and can be managed without imaging studies. Secondary (referred) otalgia is caused by pathologies in locations outside the ear and results from shared pathways of sensory innervation. ${ }^{1-7}$ Some etiologies may also be diagnosed on the basis of additional clinical findings and supported by targeted imaging (eg, temporomandibular joint or dental).

Received April 29, 2020; accepted after revision July 7.

From the Departments of Radiology (E.A., M.K., S.E.J.C.) and Otolaryngology (I.P.) Guy's and St. Thomas' Hospital, London, United Kingdom; School of Biomedical Engineering and Imaging Sciences Clinical Academic Group (I.P., S.E.J.C.) and Department of Neuroradiology (S.E.J.C.), King's College Hospital, London, United Kingdom.

Please address correspondence to Dr Steve E. J. Connor, Department of Neuroradiology, Ruskin Wing, Kings College Hospital, Denmark Hill, London SE5 9RS, United Kingdom; e-mail: steve.connor@nhs.net; @SEJConnor
Upper aerodigestive tract (UAT) malignancies are a potential cause of secondary otalgia, and it is a clinical feature that prompts rapid referral for head and neck cancer investigation in most clinical networks ${ }^{8}$; therefore, fiberoptic nasoendoscopy (FNE) is usually performed in the early diagnostic work-up in these patients. Even in the presence of a normal FNE, clinicians may remain concerned that a submucosal UAT lesion or other clinically occult pathology may be present, so cross-sectional imaging is frequently proposed. Despite this common clinical practice, to our knowledge, the diagnostic value of face and neck imaging in patients referred with otalgia has not been documented. We, therefore, performed a single-center retrospective study that analyzed the diagnostic yield, management impact, and therapeutic impact of imaging with CT and MR of the skull base, face, and neck in patients referred with otalgia but without clinically overt disease over a 9-year period.

\section{MATERIALS AND METHODS}

We performed a retrospective search of all CT and MR imaging requests on the radiology information systems, for requests and reports that contained the specific terms "otalgia," "earache," or 
“ear pain” between January 2009 and September 2017. Cases in which dedicated CT or MR imaging was performed for targeted anatomic regions (eg, skull base, intracranial, temporomandibular joint, and paranasal sinus) were excluded. Also excluded were cases in which clinical examination, including FNE, identified the cause of otalgia before imaging (eg, middle ear and external ear inflammation, foreign body in the external ear, peritonsillar abscess, palpable nodal metastases in the neck, and previously identified mandibular osteochondroma). Routine contrast-enhanced imaging protocols for $\mathrm{CT}$ and $\mathrm{MR}$ imaging encompassed the whole face and neck. The imaging was reported by dedicated head and neck radiologists.

The clinical and imaging data were interrogated by using electronic patient record, PACS, and radiology information systems. For the remaining cases, demographic information, type of imaging, laterality of otalgia, clinical examination findings (including FNE), imaging findings, subsequent clinical management, and final diagnoses were recorded. Presenting symptoms were divided into 4 categories: pure otalgia, otalgia with UAT symptoms, otalgia with other symptoms, and otalgia in patients with a history of head and neck cancer. Imaging findings were classified as important, indeterminate, or unimportant abnormality. Important abnormalities included those that may be etiologies for otalgia or that were deemed major or moderate unrelated imaging findings. ${ }^{9}$ Imaging findings that potentially cause otalgia were defined as those pathologies that occur in ipsilateral locations that are recognized to cause referred pain to the ear; these were assessed by a combined clinicoradiology team. Unrelated imaging findings were categorized as major, moderate, or minor, according to previous guidelines on incidental imaging findings from the Royal College of Radiologists. ${ }^{9}$ Major findings were defined as those that always required further investigation and were likely to have adverse health effects, whereas moderate findings were those that usually required further investigation but health effects were unclear. Minor findings were defined as those that did not require further investigations or have adverse health effects (eg, degenerative cervical spine disease, generalized or mild sinonasal or dental disease, thyroid nodules that do not require biopsy, venous malformation, and thymic hyperplasia). For the purpose of this study, minor findings with no important abnormality were grouped together for subsequent analysis of the diagnostic yield, management impact, and therapeutic impact. ${ }^{9}$

The final diagnostic outcomes were considered important if they were in 1 of the following 3 groups: UAT malignancies, new abnormalities that potentially cause ipsilateral otalgia, and other new major or moderate findings unrelated to otalgia.

Patients in whom malignancy had been identified on FNE before imaging were recorded separately because imaging was used for staging rather than diagnosis. For the remaining patients, the "diagnostic yield" (the number of studies with a new or major finding divided by the total number of studies) was used as an indicator of the value of the study in assisting diagnosis. The term "management impact" (the number of studies that result in a change in clinical management divided by the total number of studies) was used as an indicator of the influence on the patient's clinical management. ${ }^{10,11}$ The term "therapeutic impact" (the number of studies that result in subsequent directed therapy for detected pathology divided by the total number of studies) was used as an indicator of truly beneficial impact of imaging. The diagnostic yield, management impact, and therapeutic impact of CT/MR imaging of the skull base, face, and neck were calculated for the detection of UAT malignancy, abnormalities that potentially cause ipsilateral otalgia, and other major or moderate findings unrelated to otalgia.

The data were described and summarized according to each of the categories of pure otalgia, otalgia with UAT symptoms, otalgia with other symptoms, and otalgia in patients with a history of head and neck cancer. The percentage incidence of UAT malignancy, any other cause of otalgia, and other moderate and/ or major findings unrelated to otalgia were calculated for each category. The diagnostic yield, management impact, and therapeutic impact were also analyzed for each category.

\section{RESULTS}

A total of 787 relevant imaging requests were identified on the initial search. Exclusions were dedicated imaging studies (skull base, intracranial, temporomandibular joint, and paranasal sinus) $(n=516)$, patients with clinically overt disease and diagnoses on clinical examination other than FNE $(n=20)$, diagnosis of malignancy on FNE $(n=13)$, and incorrect clinical information about the presence of otalgia after review of the electronic patient record $(n=3)$.

Of the remaining 235 patients who were included in the analysis, $65 \%$ were female and $35 \%$ male patients (female to male, 152 to 83 ). The mean \pm standard deviation age at the time of imaging was $52 \pm 15.2$ years (range, 8-90 years), and this included 4 patients $<16$ years old. All were referrals from secondary care, with $89 \%$ of requests from ear, nose, and throat specialists; head and neck surgeons; or oral and maxillofacial surgeons $(n=210)$. The remaining $11 \%$ of the requests came from other specialties, such as oncology, oral medicine, audiovestibular clinic, neurology, and rheumatology $(n=25)$. With regards to imaging modality, $54 \%$ of the patients underwent MR imaging $(n=126)$ and $46 \%$ underwent CT $(n=109)$ of the face and neck.

\section{Otalgia Symptom Categories}

The study cohort was grouped into 4 main categories: pure otalgia (20\% [47/235]), otalgia with UAT symptoms (32\% [76/235]), otalgia with other symptoms (35\% [83/235]), and otalgia with a history of previous hand and neck malignancy (13\% [29/235]). Imaging was performed for bilateral otalgia in $8 \%$ (17/235), leftsided otalgia in $40 \%$ (95/235), and right-sided otalgia in $51 \%$ (120/235). No information on laterality was provided in 1\% (3/ 235). The details of associated symptoms are provided in Tables 1 and 2. FNE findings were provided in $67 \%$ of the cases (156/235) and were categorized into normal or benign, indeterminate, or diagnostic, as illustrated in Table 3.

\section{Analysis by Subgroup}

Pure Otalgia. The pure otalgia group accounted for $20 \%$ of the total study cohort (47/235). Of these, 66\% (31/47) had FNE as part of their clinical examination before imaging, which was indeterminate in 7 patients. No important abnormality was found on imaging in 98\% (46/47). In 1 patient who had indeterminate findings on initial FNE (2\%), the imaging outcome was also indeterminate 
Table 1: Otalgia with UAT symptoms-Symptom type and number

\begin{tabular}{llc}
\hline UAT Symptoms & No. & Percentage \\
\hline Total & 87 & 100 \\
Odynophagia & 64 & 74 \\
Dysphagia & 7 & 8 \\
Globus & 6 & 7 \\
Voice change & 5 & 6 \\
Nasal blockage & 2 & 2 \\
Epistaxis & 2 & 2 \\
Anosmia & 1 & 1 \\
\hline
\end{tabular}

Table 2: Otalgia with other symptoms-Symptom type and incidence

\begin{tabular}{llc}
\hline \multicolumn{1}{c}{ Other Symptoms } & No. & Percentage \\
\hline Total & 84 & 100 \\
Neck pain and/or swelling & 31 & 37 \\
Facial pain & 16 & 19 \\
Headache & 11 & 13 \\
Tinnitus & 8 & 9 \\
Hearing loss & 6 & 7 \\
Vertigo & 4 & 5 \\
Facial weakness and/or numbness & 4 & 5 \\
Trismus & 3 & 4 \\
Contralateral neck pain & 1 & 1 \\
\hline
\end{tabular}

Table 3: Categories of FNE findings

\begin{tabular}{lll}
\hline \multicolumn{1}{c}{ Benign } & \multicolumn{1}{c}{ Indeterminate } & \multicolumn{1}{c}{ Diagnostic } \\
\hline $\begin{array}{l}\text { Reinke edema, candida, } \\
\text { presbylaryngitis, interarytenoid } \\
\text { edema, laryngopharyngeal reflux, } \\
\text { rhinitis, lingual hyperplasia }\end{array}$ & $\begin{array}{l}\text { Pooling in pyriform fossae, foci } \\
\text { of soft-tissue prominence, } \\
\text { asymmetry within the } \\
\text { nasopharynx and/or larynx, } \\
\text { shallow ulceration }\end{array}$ & $\begin{array}{l}\text { Masses; ulcerated } \\
\text { lesions; fixed, } \\
\text { edematous } \\
\text { vocal cords }\end{array}$ \\
\hline
\end{tabular}

The total diagnostic yield of imaging in otalgia with UAT symptoms in the absence of overt malignancy detected on FNE, therefore, was $0 \%$ for UAT malignancy, $1 \%$ for any other cause of otalgia, and $1 \%$ for any other moderate or major findings unrelated to otalgia. With 16 patients in total undergoing further investigations based on the imaging findings, the management impact was $21 \%$, but the total therapeutic impact was $0 \%$.

Otalgia with Other Symptoms. The otalgia with other symptoms group accounted for $35 \%$ of the total study cohort (83/235). The FNE findings were normal or benign in 58\% (48/83) and indeterminate in $5 \%(4 / 83)$. There was no documentation of FNE in the other $37 \%$ (31/83). No important abnormality was found on imaging in $88 \%(73 / 83)$. Two patients were found to have UAT malignancy on imaging (2\%), neither of whom had undergone FNE as part of a clinical examination before being referred for imaging; 1 patient found to have a neck mass had refused to have FNE despite the medical recommendation, and the other patient, with trismus as an associated symptom, had been seen in a specialty setting in which FNE could not be offered as part of a clinical examination.

In another 4 patients (5\%), imaging identified the underlying cause of otalgia, which included pathologic fracture of the cervical spine, ipsilateral severe sinonasal disease, ipsilateral tonsillar enlargement (subsequently confirmed reactive hyperplasia on histology), and Warthin tumor of the parotid. In a further 3 patients (4\%), imaging detected moderate or major new findings unrelated to otalgia, including neck vein thrombosis, thyroglossal duct cyst, and calcified mediastinal thymus. In the remaining patient, the imaging outcome was indeterminate but further investigations

and repeated FNE was subsequently performed, which was normal. The diagnostic yield of head and neck imaging for those who presented with pure otalgia, therefore, was $0 \%$, and the management impact figure was $1 \%$ (normal repeated FNE), whereas the overall therapeutic impact of imaging was $0 \%$. The details of this subgroup are illustrated in On-line Fig 1.

Otalgia with UAT Symptoms. The otalgia with UAT symptoms group accounted for $32 \%$ of the total study cohort (76/235). The FNE findings were normal or benign in 67\% (51/76) and indeterminate in $20 \%(15 / 76)$. There was no documentation of FNE in the other $13 \%$ (10/76). No important abnormality was found on imaging in $79 \%$ (60/76). There was no imaging finding of UAT malignancy, whereas in 1 patient (1\%) imaging demonstrated ipsilateral tonsillar enlargement, considered to be the cause of otalgia. In another patient (1\%), imaging identified a moderate or major new finding unrelated to otalgia of a pituitary nodule, which, subsequently, was managed conservatively with MR imaging surveillance. There were 15 patients $(20 \%)$ with indeterminate imaging findings, which then led to further investigations (panendoscopy, biopsy, tonsillectomy, 11; further imaging with MR and PET, 2; fine needle aspiration cytology, 1; repeated FNE, 1), but none revealed UAT malignancy. The details of this subgroup are illustrated in On-line Fig 2. confirmed a benign pathology unrelated to otalgia (calcified thyroid nodule). All but 1 patient with indeterminate or important imaging findings had further investigations $(n=2)$, monitoring $(n=1)$, and/or surgical intervention $(n=6)$. The details of this subgroup are illustrated in On-line Fig 3.

The total diagnostic yield of imaging in otalgia with other symptoms in the absence of overt malignancy detected on FNE, therefore, was $2 \%$ for UAT malignancy, $5 \%$ for any other cause of otalgia, and $4 \%$ for any other moderate or major findings unrelated to otalgia. With 9 patients in total undergoing further investigation, monitoring, or intervention based on the imaging findings, the management impact was $11 \%$, Overall, 5 patients who presented with otalgia with other symptoms underwent definitive therapeutic interventions, which led to a total therapeutic impact figure of $6 \%$.

Otalgia with a History of Head and Neck Cancer. The otalgia with a history of head and neck cancer group accounted for $13 \%$ of the total study cohort (29/235). The FNE findings were normal or benign in $28 \%(8 / 29)$. In the other $72 \%(21 / 29)$, there was no documentation of FNE before imaging, a clear reason for this being recorded in only 3 patients (seen by a specialist unable to perform FNE, 2; no endoscope was available on day 1). No important abnormality was found on imaging in $48 \%$ (14/29). Ten 
patients were found to have UAT malignancy on imaging (34\%), 9 of whom had recurrences and the other had a new UAT primary. Of note, only 3 of these 10 patients had undergone FNE before imaging. In another patient (3\%), imaging identified mandibular osteoradionecrosis as the underlying cause of otalgia. No other moderate or major findings were identified on imaging. There were 4 patients (14\%) with indeterminate imaging findings, which then led to further investigations (further imaging with MR/ PET, 2; biopsy, 1; FNE, 1), but none revealed UAT malignancy. The details of this subgroup are illustrated in On-line Fig 4.

The total diagnostic yield of imaging in otalgia with a history of head and neck cancer, therefore, was 34\% for UAT malignancy and $3 \%$ for any other cause of otalgia. With 15 patients in total undergoing further investigations, monitoring, or interventions based on the imaging findings, the management impact was 52\%. All 10 patients with UAT malignancy detected on imaging received further treatment, which led to a total therapeutic impact figure of $34 \%$.

Summary Comparison of Significant Outcomes with and without a History of Head and Neck Cancer. The diagnostic yield of imaging for otalgia, with or without other symptoms, of patients who lacked a history of head and neck cancer, was $1 \%$ for UAT malignancy (2/206), 2\% for abnormalities related to otalgia (5/ $206)$, and $2 \%$ for other moderate or major findings (4/206). This compared with $34 \%$ for UAT malignancy (10/29), 3\% for abnormalities related to otalgia (1/29), and $0 \%$ for other moderate or major findings $(0 / 29)$ in those with a history of head and neck cancer. The therapeutic impact increased from $2 \%$ in patients without head and neck cancer $(5 / 206)$ to $34 \%$ in patients with head and neck cancer (10/29).

\section{DISCUSSION}

In almost $50 \%$ of patients, ear pain may be arising from disease distant to the ear. ${ }^{12,13}$ The mechanism of this secondary otalgia is still poorly understood. The "convergence-projection theory" is the most widely accepted potential pathophysiologic mechanism, whereby multiple nerves converge into a shared neural pathway, which results in the CNS being unable to distinguish the origin of the nociceptor stimulation. ${ }^{14}$ Sensory innervation of the peri-auricular region, external ear, and middle ear is derived from a range of cutaneous and cranial nerves, including branches of the trigeminal, facial, glossopharyngeal, and vagus nerves, as well as the cervical plexus and autonomic fibers. Sources of such referred pain, therefore, include the entire UAT, retroand parapharyngeal regions, major salivary glands, thyroid gland, teeth, sinonasal region, temporomandibular joint, cervical spine, and thorax. ${ }^{1,5,15,16}$ Although the underlying cause of secondary otalgia is benign in most cases, ${ }^{6,15,17,18}$ the possibility of severe pathology such as UAT malignancy must always be considered.

When a patient presents with otalgia in the absence of clinically overt disease, CT or MR imaging of the face and neck has been proposed to evaluate for pathologies remote to the ear. ${ }^{1,19,20}$ However, there has been no previous study that addressed the overall diagnostic yield and impact on management decisions in this clinical scenario. It, therefore, is not surprising that there are currently no national imaging guidelines for otalgia.
Furthermore, otalgia is not represented as a clinical feature in either the UK iRefer ${ }^{21}$ or the American College of Radiology Appropriateness Criteria. ${ }^{22}$ Clinical reviews on the subject vary in their emphasis of imaging and at which point in the clinical pathway it should be considered. ${ }^{19,23-26}$ Some investigators have considered imaging to be appropriate after specialist review and in the presence of "red flag" clinical features (eg, weight loss and other UAT symptoms), particularly with prolonged and unilateral symptoms, ${ }^{17,23-26}$ though others state that it would be indicated in all patients who present with otalgia and a negative ear, nose, and throat examination. ${ }^{19}$

This issue has become of increasing importance in the context of guidelines and targets for rapid imaging turnaround required for patients with suspected head and neck cancer. This approach has been supported by evidence for a shorter time to diagnosis being associated with more favorable outcomes. ${ }^{27}$ The 2005 iteration of the United Kingdom National Institute of Clinical Care Excellence guidelines ${ }^{28}$ identified unexplained otalgia with normal otoscopy as a clinical presentation that required fast-track referral. Although otalgia was removed from the subsequent, more evidence-based 2015 United Kingdom National Institute of Clinical Care Excellence guidelines, ${ }^{29}$ it remains a clinical criterion for rapid assessment within a number of clinical networks. ${ }^{8}$ A recent study of 5000 patients demonstrated a statistically significant association between prolonged otalgia with normal otoscopy and head and neck cancer, and the investigators argue for inclusion of this clinical presentation in future guidelines. The NHS England and NHS Improvement ${ }^{30} 28$-day faster diagnosis standard will further increase pressures on the imaging department to rapidly image and report for patients referred on these pathways.

Previous studies have addressed the final presumptive diagnosis in patients with otalgia and normal otoscopy being referred for specialist evaluation, with the subsequent diagnostic yield for UAT malignancy varying between $6 \%$ and $18 \% .{ }^{15,31}$ However, it should be borne in mind that these figures represented the overall incidence of UAT malignancy and did not describe what proportion of the cancer diagnoses was made on imaging rather than on clinical assessment, including FNE. Because clinical pathways usually indicate that full specialist examination should be performed before imaging, what has yet to be elucidated is the true value of imaging of the face and neck in patients who present with otalgia but a normal clinical examination. Although a single case series has described infratemporal fossa malignancy in $2 \%$ of patients who presented with otalgia and normal ear, nose, and throat examination, ${ }^{20}$ there has been no systematic approach to the imaging yield for all pathologies.

To the best of our knowledge, our study is the first to address the question of appropriateness of CT and MR imaging of the face and neck in patients with otalgia in the absence of clinically overt disease. Our results show that the contribution of imaging is negligible in those who present with pure otalgia, no other associated symptoms, and no history of head and neck cancer. The diagnostic yield and therapeutic impact of imaging are similarly very low in cases of otalgia with associated UAT or other symptoms, especially when there is no important finding on FNE. The situation in which imaging plays a significant role seems to be in the context of otalgia in patients with a history of 
head and neck cancer. However, the figures obtained from our study cohort should be interpreted with caution, given the low rates of FNE being performed before referral for imaging. It may be that, if FNE had been carried out in all the cases, then the proportion of UAT malignancy detected on imaging because of new findings would have been lower. It was noted that a high proportion of patients (62\%) with previous head and neck cancer proceeded straight to imaging without a clear reason for the lack of documented FNE outcomes. Clinicians should be reminded that, especially in this high-risk group, imaging should complement clinical findings rather than replace them.

Another area that is worth further discussion is how to interpret the management impact of imaging. Although it would seem at first glance that imaging had a relatively significant management impact in the otalgia with UAT symptoms group (21\%), every patient in this group proceeded to further investigations on the basis of indeterminate imaging findings, with an ultimate diagnostic yield and therapeutic impact figures of $0 \%$ and $1 \%$, respectively. The incidence of unrelated or "incidental" imaging findings in our cohort is not an unexpected phenomenon. Incidental findings have been found to be common in healthy volunteers in research studies, being documented in 3\%-12\% of neuroimaging studies and 30\% of body imaging studies. ${ }^{9}$

Incidentally detected pathologies may become more prevalent relative to the symptomatic pathologies when there is a lower threshold for imaging patients. The implications of such discoveries will not always be positive and may cause the patient unnecessary anxiety. Furthermore, asymptomatic incidental abnormalities may trigger further investigations with potential risks and financial costs but without clear clinical benefit. We, therefore, would propose that, when evaluating increased requirements for imaging services, management impact figures should be analyzed not in isolation but in conjunction with diagnostic yields and therapeutic impact.

It is recognized that there are additional potential drivers and perceived benefits of imaging patients with otalgia that extend beyond the diagnostic yield. Such factors may relate to the clinician's intolerance of uncertainty, fear of legal action, or perception that imaging is required to complete the patient episode. Patient expectation and the need for reassurance may also result in increased imaging requests. However, a systematic review of 5 randomized controlled trials, which included $>1500$ patients, showed little value of imaging in the reassurance of patients in primary care. ${ }^{32}$ The lack of data on the diagnostic yield of imaging in otalgia without clinically overt disease, combined with the aforementioned factors and cancer imaging targets, has led to increased demand on imaging services in recent years. It is hoped that the outcomes from our study will help establish a more evidence-based approach and efficient use of resources.

\section{Limitations}

We acknowledge that our study had a number of limitations. First, the retrospective design and the lack of current imaging guidelines for patients with otalgia led to some heterogeneity of the data available and likely variation in the approach to imaging these patients. Potential bias may have been introduced by the retrospective selection of the study group. Second, we did not follow those patients with otalgia and no clinically overt disease who had not undergone imaging. Third, in excluding cases of targeted imaging studies, we made an assumption that they had specific clinical features of skull base, intracranial, temporomandibular joint, or paranasal sinus disease. Fourth, the variable use of either CT or MR imaging also may have led to bias because the imaging modalities vary in their diagnostic strengths; however, the dataset was equally divided between the 2 imaging modalities. Fifth, the availability of data on FNE findings before imaging was inconsistent, but we aimed to overcome this shortcoming by analyzing the documentation of imaging outcomes in both those patients with and without FNE having been performed.

\section{CONCLUSIONS}

To our knowledge, our study is the first to address the diagnostic yield, management impact, and therapeutic impact of imaging in otalgia without clinically overt disease. The diagnostic yield of imaging was extremely low for otalgia, with and without associated symptoms, but increased markedly when there was a history of head and neck cancer. Similarly, the therapeutic impact is very limited unless there is a history of head and neck cancer or when FNE cannot be performed. Analysis of this data will help rationalize imaging guidelines for patients with otalgia in the context of increasing demands on imaging services.

Disclosures: Steve E. J. Connor-UNRELATED: Grants/Grants Pending: Guy's and St Thomas Charity, Comments: Grant awarded 2012 (last payment $>24$ months ago) for a research project on the use of DWI MRI in head and neck cancer follow up.* *Money paid to the institution.

\section{REFERENCES}

1. Chen RC, Khorsandi AS, Shatzkes DR, et al. The radiology of referred otalgia. AJNR Am J Neuroradiol 2009;30:1817-23 CrossRef Medline

2. Ely J, Hansen MR, Clark EC. Diagnosis of ear pain. Am Fam Physician 2008;77:621-28 Medline

3. Greenall C, Rhys R. Radiology of Referred Otalgia. ENT and Audiology News 2009;23:37-40

4. Kuttila SJ, Kuttila MH, Niemi PM, et al. Secondary otalgia in an adult population. Arch Otolaryngol Head Neck Surg 2001;127:401-05 CrossRef Medline

5. Weissman JL. A pain in the ear: the radiology of otalgia. AJNR Am J Neuroradiol 1997;18:1641-51 Medline

6. Jaber J, Leonetti J, Lawrason A, et al. Cervical spine causes for referred otalgia. Otolaryngol Head Neck Surg 2008;138:479-85 CrossRef Medline

7. Hersh S, Hersh JN. Referred otalgia: a diagnostic conundrum in an aging population. Consultant 2005;55:516-23

8. Pan-London Suspected Cancer Referral Guide - Head \& Neck. https://1-nelh-uks-wp05-wa.azurewebsites.net/wp-content/uploads/ 2019/04/Pan-London-Suspected-Cancer-Referral-Guide-Head-andNeck.pdf. Accessed November 24, 2019

9. The Royal College of Radiologists. Management of Incidental Findings Detected During Research Imaging. London: The Royal College of Radiologists; 2011

10. Fakhran S, Alhilali L, Branstetter BF IV. Yield of CT angiography and contrast-enhanced MR imaging in patients with dizziness. AJNR Am J Neuroradiol 2013;34:1077-81 CrossRef Medline

11. Loop J, Lusted LE. American College of Radiology diagnostic efficacy studies. AJR Am J Roentgenol 1978;131:173-79 CrossRef Medline

12. Yanagisawa K, Kveton JF. Referred otalgia. Am J Otolaryngol 1992;13:323-27 CrossRef Medline

13. Thaller S, De Silva A. Otalgia with a normal ear. Am Fam Physician 1987;36:129-36 Medline 
14. Wright EF. Referred craniofacial pain patterns in patients with temporomandibular disorder. J Am Dent Assoc 2000;131:1307-15 CrossRef Medline

15. Fenton JE, Uzomefuna V, O'Rourke C, et al. Applying the Ts of referred otalgia to a cohort of $\mathbf{2 2 6}$ patients. Clin Otolaryngol 2018;43:937-40 CrossRef Medline

16. Scarbrough T, Day T, Williams T, et al. Referred otalgia in head and neck cancer. Am J Clin Oncol 2003;26:e157-62 CrossRef Medline

17. Rerucha $\mathrm{C}$. What is the differential diagnosis of otalgia in an adult with a normal ear examination? Evidence-Based Practice 2016;19:1516 CrossRef

18. Al-Sheikhli A. Pain in the ear-with special reference to referred pain. J Laryngol Otol 1980;94:1433-40 CrossRef Medline

19. Visvanathan V, Kelly G. 12 minute consultation: an evidence-based management of referred otalgia. Clin Otolaryngol 2010;35:409-14 CrossRef Medline

20. Leonetti J, Li J, Smith PG. Otalgia. An isolated symptom of malignant infratemporal tumors. Am J Otol 1998;19:496-98 Medline

21. RCR iRefer guidelines. https://www.rcr.ac.uk/clinical-radiology/ being-consultant/rcr-referral-guidelines/about-irefer/irefer-england. Accessed January 10, 2020

22. American College of Radiology ACR Appropriateness Criteria. https:// acsearch.acr.org/docs/69509/Narrative. Accessed January 10, 2020

23. Harrison E, Cronin M. Otalgia. Aust Fam Physician 2016;45:493-97 Medline

24. Charlett S, Coatesworth A. Referred otalgia: a structured approach to diagnosis and treatment. Int J Clin Pract 2007;61:1015-21 CrossRef Medline
25. Earwood J, Rogers T, Rathjen N. Ear pain: diagnosing common and uncommon causes. Am Fam Physician 2018;97:20-27 Medline

26. Finnikin S, Mitchell-Innes A. Recurrent otalgia in adults. $B M J$ 2016;354:i3917 CrossRef Medline

27. Neal $\mathrm{RD}$, Tharmanathan $\mathrm{P}$, France $\mathrm{B}$, et al. Is increased time to diagnosis and treatment in symptomatic cancer associated with poorer outcomes? Systematic review. Br J Cancer 2015;112(suppl 1):S92-107 CrossRef Medline

28. National Institute for Health and Care Excellence. Referral Guidelines for Suspected Cancer. London: National Institute for Health and Care Excellence; 2005. http://www.nice.org.uk/guidance/cg27chapter/ guidance\#head-and-neck-cancer-including-thyroid-cancer. Accessed January 10, 2020

29. National Institute for Health and Care Excellence. Referral Guidelines for Suspected Cancer. London: National Institute for Health and Care Excellence; 2015. https://www.nice.org.uk/guidance/ng12/chapter/1Recommendations-organised-by-site-of-cancer\#head-and-neck-cancers. Accessed January 10, 2020

30. NHS England and NHS Improvement. Diagnosing cancer earlier and faster. https://www.england.nhs.uk/cancer/early-diagnosis/ Accessed January 10, 2020

31. Tikka T, Pracy P, Paleri V. Refining the head and neck cance referral guidelines: a two-centre analysis of 4715 referrals. Clin Otolaryngol 2016;41:66-75 CrossRef

32. van Ravesteijn $H$, van Dijk I, Darmon $D$, et al. The reassuring value of diagnostic tests: a systematic review. Patient Educ Couns 2012;86:3-8 CrossRef Medline 